\title{
Tobacco plastidial thioredoxins as modulators of recombinant protein production in transgenic chloroplasts
}

\author{
Ruth Sanz-Barrio ${ }^{1, \dagger}$, Alicia Fernández-San Millán ${ }^{1, \dagger}$, Patricia Corral-Martínez ${ }^{2}$, José M. Seguí-Simarro² and \\ Inmaculada Farran ${ }^{1, *}$ \\ ${ }^{1}$ Instituto de Agrobiotecnología (UPNA-CSIC-Gobierno de Navarra), Pamplona, Spain \\ ${ }^{2}$ Instituto para la Conservación y Mejora de la Agrodiversidad Valenciana (COMAV), Universidad Politécnica de Valencia, Ciudad Politécnica de la Innovación, \\ Valencia, Spain
}

Received 15 October 2010;

revised 31 January 2011;

accepted 31 January 2011.

*Correspondence (Tel 34948 168034; fax 34948 232191; email farran@unavarra.es) ${ }^{\dagger}$ Both authors contributed equally to this work.

Keywords: thioredoxin, plastid transformation, human serum albumin, tobacco.

\begin{abstract}
Summary
Thioredoxins (Trxs) are small ubiquitous disulphide proteins widely known to enhance expression and solubility of recombinant proteins in microbial expression systems. Given the common evolutionary heritage of chloroplasts and bacteria, we attempted to analyse whether plastid Trxs could also act as modulators of recombinant protein expression in transgenic chloroplasts. For that purpose, two tobacco Trxs ( $m$ and $f$ ) with different phylogenetic origins were assessed. Using plastid transformation, we assayed two strategies: the fusion and the co-expression of Trxs with human serum albumin (HSA), which was previously observed to form large protein bodies in tobacco chloroplasts. Our results indicate that both Trxs behave similarly as regards HSA accumulation, although they act differently when fused or coexpressed with HSA. Trxs-HSA fusions markedly increased the final yield of HSA (up to $26 \%$ of total protein) when compared with control lines that only expressed HSA; this increase was mainly caused by higher HSA stability of the fused proteins. However, the fusion strategy failed to prevent the formation of protein bodies within chloroplasts. On the other hand, the co-expression constructs gave rise to an absence of large protein bodies although no more soluble HSA was accumulated. In these plants, electron micrographs showed HSA and Trxs co-localization in small protein bodies with fibrillar texture, suggesting a possible influence of Trxs on HSA solubilization. Moreover, the in vitro chaperone activity of Trx $m$ and $f$ was demonstrated, which supports the hypothesis of a direct relationship between Trx presence and HSA aggregates solubilization in plants co-expressing both proteins.
\end{abstract}

\section{Introduction}

Thioredoxins (Trxs) are small, heat-stable proteins (c. 12$14 \mathrm{kDa})$, found in all free-living organisms, that catalyse redox reactions, promote disulphide bond formation and synergistically work with protein disulphide isomerases and/or chaperones to control a wide range of biochemical pathways (Buchanan and Balmer, 2005; Berndt et al., 2008). Because of their solubility, stability, small size, globular compact structure and high translatability, Trxs have been broadly used in biotechnology. In particular, the Escherichia coli TrxA has been employed in microbial systems as a solubility and stability enhancer of recombinant proteins that cannot normally form disulphide bridges (LaVallie et al., 2000; Kolaj et al., 2009). A number of examples of Trx co-expression exist in the literature; this system produces the recombinant protein in a free form and does not require a subsequent processing with specific peptidases. TrxA co-production has been demonstrated to markedly enhance the amount of active proteins (Bessette et al., 1999; Garcia-Ortega et al., 2000; Yuan et al., 2004) and dramatically increase the solubility of proteins that otherwise produce inclusion bodies (IBs) (Yasukawa et al., 1995). TrxA has also been successfully used as a fusion partner to avoid IB formation and increase the level of soluble proteins heterologously expressed in E. coli (LaVallie et al., 1993, 2000, 2003; LaVallie and McCoy, 1995; Ribas et al., 2000; Jurado et al., 2006; Xu et al., 2006, 2007; Bogomolovas et al., 2009). Other studies have compared the Trxs behaviour in fusion or co-expression assays and concluded that they have different effects on recombinant protein solubility (Yuan et al., 2004). Although several hypothesis have tried to explain the role of Trxs with recombinant proteins (LaVallie et al., 2000, 2003; Kumar et al., 2004), to date, the mechanisms of how Trx achieves enhanced expression and/or chaperone activity are poorly understood (Bogomolovas et al., 2009).

Because of the endosymbiotic origin of plastids as well as the prokaryotic-like structure and metabolism (McFadden, 2001), such Trx properties in microbial organisms might also be useful for the chloroplast expression system. Chloroplasts are currently known to contain five typical Trx isoforms: $f, m, x, y$ and $z$. The $m$ - and f-types act as messengers in the ferredoxin/Trx system by transmitting the redox signal to target enzymes. Trx $f$ is mainly implicated in the redox regulation of photosynthetic carbon assimilation, whereas m-type Trxs might also play additional roles (Issakidis-Bourguet et al., 2001; Meyer et al., 2005). The $x$ - and $y$-types do not appear to primarily be involved in enzyme regulation, but are rather implicated in stress response (reviewed in Schurmann and Buchanan, 2008). The recently reported Trx z seems to have an important role in the regulation of plastid-encoded polymerase-dependent transcription in 
chloroplasts (Arsova et al., 2010). Although all plastidial Trxs are codified in the nucleus and their overall structure is similar, phylogenetic studies and structural comparisons have demonstrated that $\operatorname{Trx} \mathrm{m}, \mathrm{x}, \mathrm{y}$ and $\mathrm{z}$ have a prokaryotic origin (Meyer et al., 2005; Arsova et al., 2010). Trx f, however, displays homology with Trxs of eukaryotic origin (Sahrawy et al., 1996). To study a putative biotechnological application of Trxs in plastids, it would be useful to assay Trxs of both prokaryotic and eukaryotic origin. In this sense, Trx $m$ and $f$ might be good candidates because they are reported to have the widest range of functions and target proteins (Lemaire et al., 2007).

A potentially ideal way to study plastid Trxs $m$ and $f$ as modulators of recombinant protein expression in plants is through plastid transformation technology. As mentioned previously, plastids are prokaryotic-like compartments where a number of homologies with microbial systems have been found. In addition, similar to bacterial fermenter technology, chloroplast transformation allows very high levels of recombinant protein expression. Average accumulation levels are in the range of $4 \%-20 \%$ of total soluble protein (Koop et al., 2007), with maximum values of $70 \%$ in the case of lysin expression (Oey et al., 2009). Studies in this field indicate that transgenic plants can produce recombinant proteins 10-100 times cheaper than cell culture systems (Mison and Curling, 2000). Moreover, to our knowledge, no information has yet been reported about how to enhance solubility and proper folding of heterologous proteins expressed in chloroplasts. Even though it has been demonstrated that chloroplasts are able to correctly fold proteins with disulphide bonds (Staub et al., 2000; Daniell et al., 2001; Molina et al., 2004; Ruhlman et al., 2007; Farran et al., 2008), recombinant proteins are not always biologically active nor accumulate in the soluble fraction (Fernandez-San Millan et al., 2003, 2008; Ruhlman et al., 2007, 2010; Daniell et al., 2009). The most useful alternative to increase the level of expression in the chloroplast transformation system is the fusion of the amino-terminus of the coding region with some downstream sequences or even entire proteins, such as GFP or GUS (Ye et al., 2001; Leelavathi and Reddy, 2003; Molina et al., 2004; Herz et al., 2005; Farran et al., 2010; Lee et al., 2010; Lentz et al., 2010). However, it may be necessary to eliminate such fusion partners by in vitro protease digestions, which would increase the final cost of production. In these cases, a co-expression system with a protein that enhances and maximizes the active soluble fraction of recombinant proteins accumulated in chloroplasts would be ideal.

We previously showed the plastidial expression of human serum albumin (HSA) (Fernandez-San Millan et al., 2003). Although it was expressed at relatively high levels in tobacco leaves, HSA accumulated as protein IBs within chloroplasts. The formation of IBs might be thought of as 'inappropriate' protein-protein interactions because of the lack of proper polypeptide folding (Mitraki and King, 1989). HSA has a complex globular structure with 17 disulphide bonds, and mismatching of cysteines might cause the insolubility of chloroplast-produced HSA. To obtain HSA with commercial characteristics, it would be necessary to include denaturation and renaturation steps in the production process, which would markedly increase the final cost. In addition, it was observed that, although HSA was present mainly as IBS, its half-life was relatively low, approximately $4 \mathrm{~h}$ (Fernandez-San Millan et al., 2007). The lack of stability and solubility of HSA encouraged us to use it as a model protein to investigate whether we could improve its production using a Trx N-terminal protein fusion or co-expression strategy.

In this work, we have explored the possible use of plant Trxs as enhancer elements of recombinant protein accumulation. To date, it has not been demonstrated that Trxs can be used for that purpose in the chloroplast transgenic expression system. Therefore, we have studied the effect of fusion and co-expression of Trx $m$ and $f$ from tobacco plastids as modulators of HSA production.

\section{Results}

\section{Design of plasmids for HSA fusion or co-expression with tobacco thioredoxins $m$ and $f$}

Tobacco Trx $m$ and $f$ genes were selected for the study of improvement in HSA expression within chloroplasts because of their plastidial localization, wide range of functions and different phylogenetic origin. Whereas Trx $f$ is of eukaryotic origin, $\operatorname{Trx} m$ displays homologies with bacterial $\operatorname{Trx} A$, which is the preferred partner to increase solubility and expression of recombinant proteins in microbial systems (Hammarstrom et al., 2002; Bogomolovas et al., 2009; Kolaj et al., 2009). To analyse whether plastidial Trxs could modulate the expression of recombinant proteins in chloroplasts, both the fusion and the co-expression of Trx $m$ and $f$ with HSA were assessed. For the fusion constructs, Trx sequences corresponding to the mature peptides were translationally fused to the HSA sequence (Figure 1a). This fusion included the flexible hinge tetrapeptide GPGP in the middle of both sequences. This tetrapeptide may reduce steric hindrance between Trx and HSA and thus facilitate HSA assembly, as has been previously observed (Molina et al., 2004). The fusions were expressed from the tobacco psbA promoter and untranslated region (UTR) and introduced into the chloroplast transformation pLD vector. The pLD vector was previously used for the expression of HSA (hereafter referred to as free-HSA), which was demonstrated to form IBs within chloroplasts in a previous work (Fernandez-San Millan et al., 2003). The pLD vector integrates genes of interest into the plastid genome between the $\operatorname{trnl}$ and $\operatorname{trn} A$ sequences (Figure 1a). This vector also includes the aminoglycoside 3 'adenylyltransferase (aadA) gene from E. coli, which confers resistance to both spectinomycin and streptomycin and is driven by the constitutive promoter of the rRNA operon (Prrn), and a multiple cloning site between the end of the $\operatorname{ard} A$ coding sequence and the psbA terminator.

For co-expression vectors, HSA and Trxs were expressed in a free form using different promoters to avoid possible deletions via homologous recombination (lamtham and Day, 2000) (Figure 1a). The design of co-expression vectors was chosen to minimize changes of the original pLD-psbAHSA (Fernandez-San Millan et al., 2003), where free-HSA accumulates to a level of $7 \%$ of total protein (TP) under regular light conditions. Trx genes were expressed from the constitutive tobacco rrn promoter followed by the T7 phage gene 10 leader sequence (PrrnG10L), which is one of the strongest known expression signals in plastids (Kuroda and Maliga, 2001a). The PrrnG10LTrxm/f cassette was introduced into the pLD-psbAHSA vector downstream of the HSA gene, which was driven by the tobacco psbA promoter and 5'-UTR.

The final vectors were sequenced, and Western blot analyses of sonicated $E$. coli extracts showed the expression of both HSA 
(a)

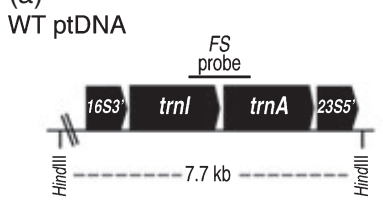

Trxs-HSA fusion ptDNA

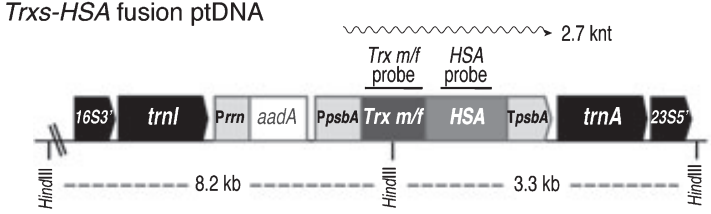

HSA/Trxs co-expression ptDNA

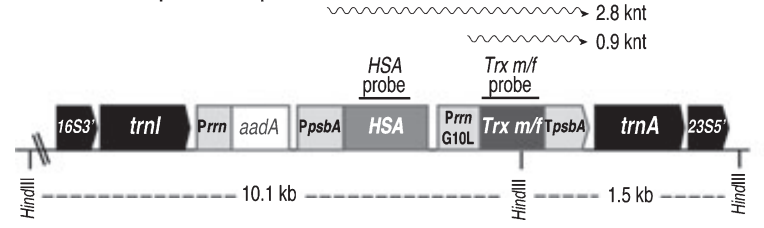

(b)

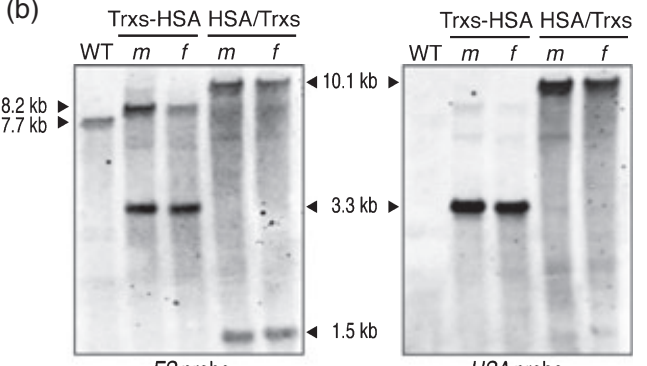

FS probe

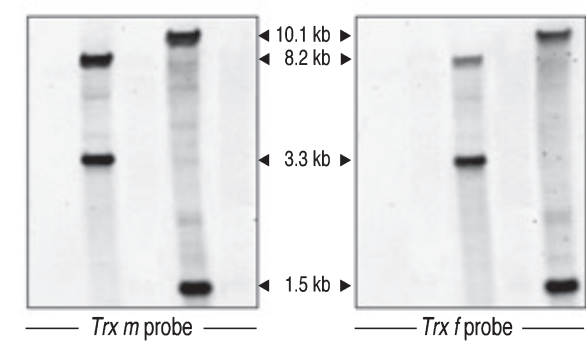

Figure 1 Integration of Trx $m / f$ and HSA genes by fusion or co-expression into the plastid genome and homoplasmy verification. (a) Map of the WT, Trxs-HSA fusion and HSA/Trxs co-expression transformed plastid genomes. The transgenes are targeted to the intergenic region between trnl and $\operatorname{trn} A$. The selectable marker gene aadA is driven by the ribosomal RNA operon promoter (Prrn). The Trxs-HSA fusion is driven by the psbA promoter and untranslated region (UTR). In co-expression, HSA is also driven by the psbA leader region followed by the PrrnG10L and the $\operatorname{Trx} m$ or $f$ sequences. Probes for the Southern blot are shown over the corresponding sequences. Mono- and dicistronic transcripts are denoted by zigzag arrows, and the expected sizes for transcripts are indicated. (b) The Southern blot analysis for the different transgenic lines is shown. The same blot was probed with FS probe to study the homoplasmic state and with HSA-, Trx m- and Trx f-specific probes. One line per construct and a WT control are shown. 16S, 23S, trnl, trnA: original sequences of the chloroplast genome; aadA: aminoglycoside 3'-adenylyltransferase; Prrn: 16S rRNA promoter; PpsbA: psbA promoter and 5'-UTR; PrrnG10L: $16 \mathrm{~S}$ rRNA promoter and the gene 10 leader from phage T7; 3'psbA: terminator region of the psbA gene; WT: wild-type; Trxs-HSA: fusion plants; HSA/Trxs: co-expression plants. Trx, thioredoxin; HSA, human serum albumin.

and Trxs, demonstrating that all four chloroplast transformation vectors are functional (data not shown).

\section{Tobacco chloroplast transformation and homoplasmic plants selection}

Chloroplast transformation by particle bombardment (Daniell, 1997) of tobacco leaves followed by selection through spectinomycin-containing plant regeneration medium produced several fusion and co-expression transformed lines (hereafter referred to as Trx-HSA and HSA/Trx, respectively), as was confirmed by PCR analysis (data not shown). Southern blot analysis was performed to verify site-specific integration and to confirm homoplasmy. Total plant DNA was digested with HindIII. The flanking sequence probe (FS probe) identified a 7.7-kb fragment in the wild-type plant, 8.2- and 3.3-kb fragments in plants expressing the Trxs-HSA fusions and 10.1 - plus 1.5-kb fragments in plants co-expressing Trxs and HSA (Figure 1a,b). The absence of 7.7$\mathrm{kb}$ bands in the transformed lines indicated homoplasmy. Absence of residual wild-type copies of the highly polyploidy plastid genome was further confirmed by maternal inheritance of the antibiotic resistance and lack of phenotypic segregation in inheritance assays (data not shown). To confirm that transgenic plants contained the $\operatorname{Tr} \mathrm{m} / \mathrm{f}$ and HSA sequences, the same membranes were stripped and probed again with the gene-specific probes (Figure 1a). Hybridization was observed in all transgenic lines and was absent in the wild-type control plant (Figure 1b). Under our growth conditions, all transplastomic lines displayed a standard phenotype with the exception of HSA/Trx $m$ co-expressing plants, which exhibited a pale-green coloration but were otherwise normal in growth and development.

\section{Thioredoxin $m$ or $f$ fusions increase HSA accumulation in transgenic chloroplasts}

Greenhouse plants from the $T_{1}$ generation of transgenic lines and free-HSA expressing plants used as control were analysed for HSA and Trx $\mathrm{m} / \mathrm{f}$ accumulation in chloroplasts. TP from mature leaves was analysed by sodium dodecyl sulphate-polyacrylamide gel electrophoresis (SDS-PAGE) and by Western blot with an anti-HSA antibody (Figure 2a). Monomers of HSA protein were detected at $66 \mathrm{kDa}$ in free-HSA and HSA/Trxs co-expressing lines. Likewise, the monomers corresponding to the Trxs-HSA fusion proteins $(80 \mathrm{kDa})$ were also detected. As was observed in free-HSA expressing plants, there were significant amounts of large and small immunoreactive bands in Trxs-HSA fusion-expressing plants. This finding suggests not only the formation of aggregates inside transgenic chloroplasts but also a certain degree of recombinant protein turnover. However, plants co-expressing HSA and either Trx m or Trx $f$ showed mainly the monomeric HSA (Figure 2a). There was neither significant aggregation nor degradation bands, although fivefold more TP was loaded. These results indicate that Trxfused HSA plants accumulated much higher levels of recombinant protein than did co-expressed HSA plants. To further assess this observation, we analysed electrophoretically separated TP samples by Coomassie staining (Figure 2b). HSA was barely detectable in Trx co-expression lines; however, a clear band was visible in both Trxs-HSA fusion lines, which was even 
(a)

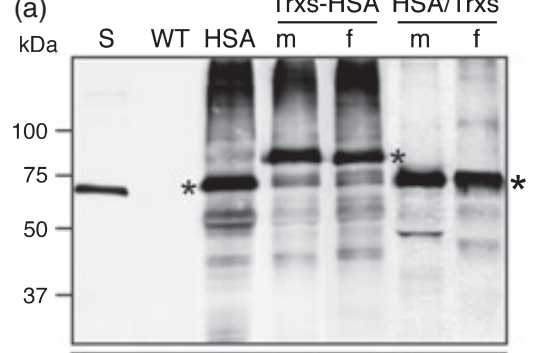

(c)

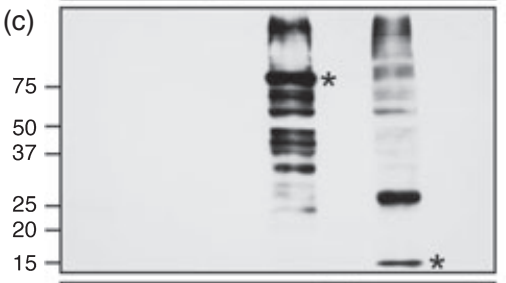

(d)

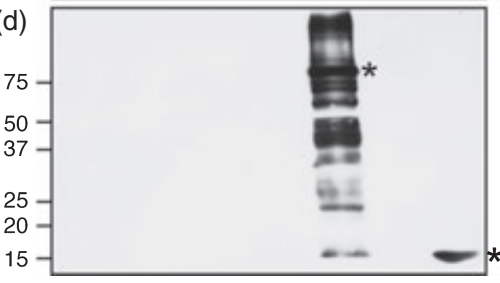

(b)

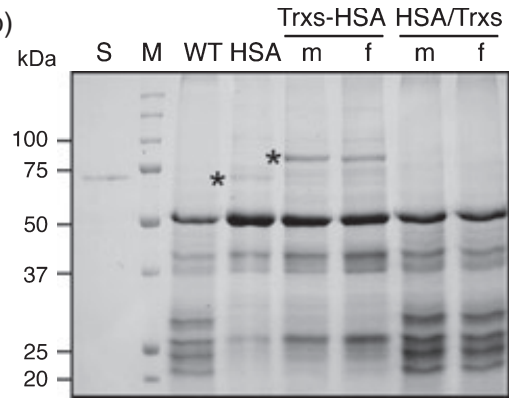

(e)

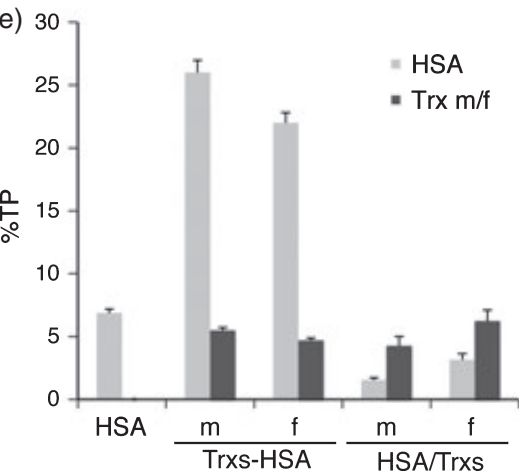

Figure 2 Characterization of HSA and Trx $\mathrm{m} / \mathrm{f}$ accumulation in fusion or co-expression plants. (a) Immunoblot analysis of protein extracts from mature leaves (fifth from top) of the different transgenic or WT lines with anti-HSA antibody. A total of $50 \mathrm{ng}$ of pure HSA were used as standard. Protein extracts of 45-day-old transgenic and WT plants were loaded onto an $8 \%$ sodium dodecyl sulphate-polyacrylamide gel electrophoresis (SDS-PAGE) gel. For free-HSA control and Trxs-HSA fused lines, proteins extracted from 0.5 and $0.2 \mathrm{mg}$ of leaves were analysed, respectively. For WT and HSA/Trxs co-expression plants, proteins extracted from $5 \mathrm{mg}$ of leaves were loaded. (b) Twenty micrograms of total protein (TP) extracted from WT and transplastomic lines were loaded onto an 8\% SDS-PAGE gel and stained with Coomassie Brilliant Blue. (c,d) The same protein extracts as for antiHSA Western blot were analysed with anti-Trx $m$ and $f$ antibodies respectively in a $13 \%$ SDS-PAGE gel. (e) HSA and Trx m/f quantification in mature leaves from transgenic tobacco plants by Western blot analysis. Results are the mean \pm SE of two measurements for two independent transgenic lines per construct and are shown as percentage of TP. S: standard; WT: wild-type plant; HSA: free-HSA control plant; Trxs-HSA: fusion plants; HSA/Trxs: co-expression plants; M: molecular weight marker. Monomers of each recombinant protein are denoted by an asterisk. Trx, thioredoxin; HSA, human serum albumin.

more abundant than in free-HSA expressing plants. Transgenic lines were also analysed by Western blot with anti-Trx $\mathrm{m} / \mathrm{f}$ antibodies (Figure $2 \mathrm{c}, \mathrm{d}$ ). As expected, aggregation and degradation bands were also present in fusion lines. Endogenous Trx $m$ and $f$ were not detected in wild-type plants, which indicated lower expression levels than in transplastomic lines. To corroborate differences in recombinant protein expression among different lines, the levels of HSA and Trxs accumulation in mature leaves (fifth from top) of greenhouse-grown plants 45 days after transplanting were determined by Western blot quantification. This technique allowed us to quantify the aggregates (bands above the expected band size) and discard the degradation bands (below the expected band size). Using serial dilutions of leaf extracts, the intensity of the immunoreacted bands corresponding to recombinant protein monomers plus aggregates was compared with that of commercial HSA standard, as explained in the experimental procedures. Accordingly, transplastomic Trx m-HSA and Trx f-HSA fusion lines accumulated recombinant HSA at levels of $26 \%$ and $22 \%$ of TP, respectively (Figure 2e). These values include not only the monomeric band detected in the Coomassie stained gel (Figure 2b) but also the abundant aggregates visible in the Western blot (Figure 2c), which explains the differences between band intensities in the Coomassie staining and quantification results. The recombinant protein levels achieved in the Trx-HSA fusion lines are approximately 3.8- and 3.2-fold higher than HSA quantified in free-HSA expressing plants (6.8\% of TP). In contrast, HSA/Trxs co-expressing plants accumulated recombinant HSA at 1.5\% and $3.1 \%$ of TP (Figure 2e) when Trx $m$ or $f$ were used, respectively. The accumulation of Trxs in co-expression lines was also quantified by Western blot (Figure 2e), whereas in fusion lines, Trx accumulation was proportionally estimated from the fusion protein amount, according to its molecular weight ( $14 \mathrm{kDa}$ for Trxs and $80 \mathrm{kDa}$ for Trxs-HSA fusion). No important differences were found among all transgenic lines, where Trx levels ranged from $4.2 \%$ to $6.2 \%$ of TP.

\section{Differences in HSA accumulation among transgenic lines are attributed to post-transcriptional factors}

Northern blot analysis was performed to determine whether or not differences in HSA and Trxs mRNA accumulation could account for the remarkable changes in HSA expression among transplastomic lines. The HSA coding region probe showed that transcripts from the psbA promoter in both fusions (2.7 knt) or co-expressions (2.8 knt) were slightly less abundant than those in free-HSA (2.3 knt) expressing plants (Figure 3a, upper panels), probably because of lower stability. It is interesting to note that such differences are in contrast to the 3.5-fold increase on 


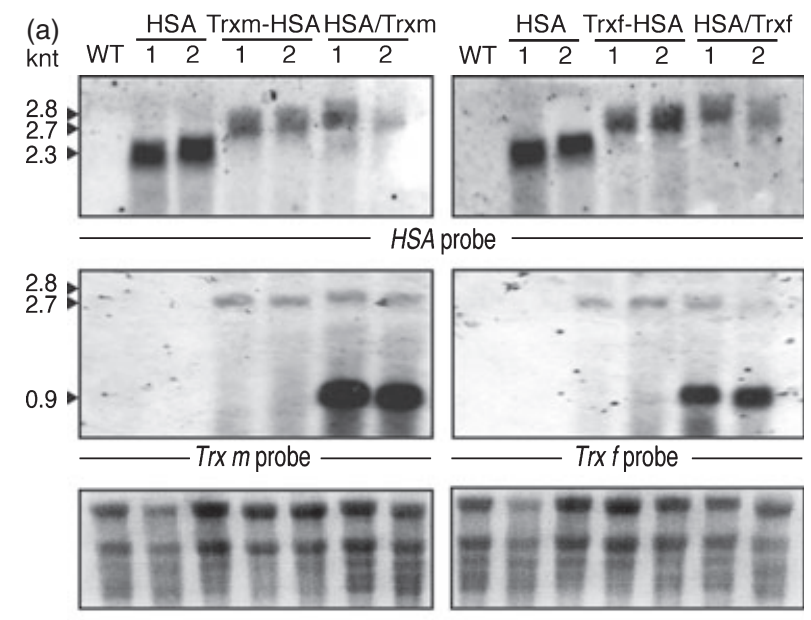

(b)

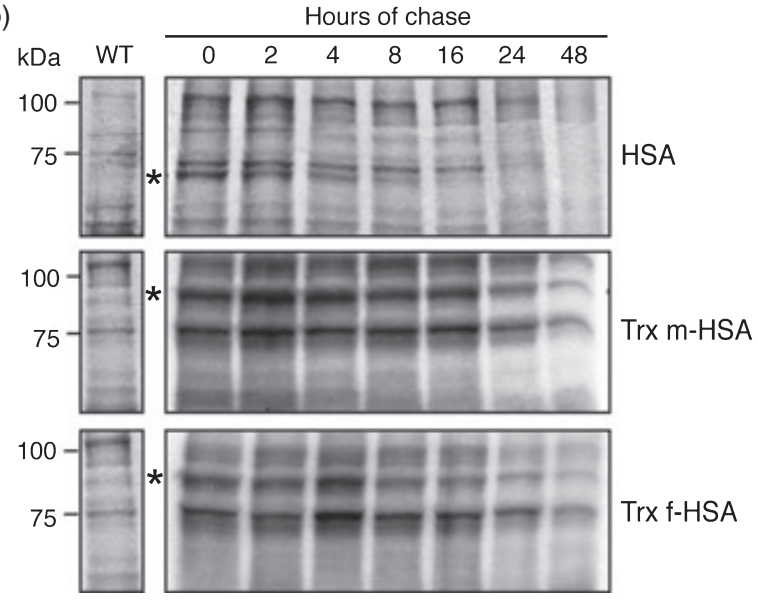

Figure 3 (a) Transcription analysis in transgenic lines. Northern blot analysis of total RNA isolated from transplastomic and WT plants. Ten micrograms of RNA were electrophoresed in denaturing conditions, blotted onto a nylon membrane and hybridized with HSA-, Trx m- or Trx $f$-specific probes. Two plants per transplastomic line are shown. As load controls, ethidium bromide-stained total leaf RNAs are reported in the lower panels. Mono- and dicistronic transcript sizes are indicated as described in Figure 1a. (b) Pulse-chase analysis of control free-HSA or Trxs-HSA fused proteins. Leaf discs were pulse-labelled for $1 \mathrm{~h}$ with ${ }^{35}$ S-Met and ${ }^{35}$ S-Cys and chased for 0, 2, 4, 8, 16, 24 and $48 \mathrm{~h}$ as indicated. After total protein extraction, analysis was performed by sodium dodecyl sulphate-polyacrylamide gel electrophoresis and autoradiography. Bands corresponding to the labelled recombinant proteins are denoted by an asterisk. As negative control, leaf discs from WT plants were also labelled. WT: wild-type plant; HSA: free-HSA control plant; Trxm-HSA and Trxf-HSA: fusion plants; HSA/Trxm and HSA/Trxf: co-expression plants. Trx, thioredoxin; HSA, human serum albumin.

average in HSA accumulation in Trxs-HSA fusion plants. The same blots were hybridized against Trx $m$ - and $f$-specific probes (Figure 3a, middle panels). In both cases, the monocistronic $\operatorname{Tr} x$ transcript $(0.9 \mathrm{knt})$ produced from the PrrnG10L promoter in co-expression lines was much more abundant than the dicistronic transcript $(2.8 \mathrm{knt})$, which includes HSA and Trx sequences. The increase in mRNA levels from this promoter may be caused by an increase in either the transcription rate or mRNA stability. However, these higher levels of monocistronic Trxs transcripts in co-expression lines did not correlate with an enhancement in Trx accumulation (Figure 2e). In no case, the

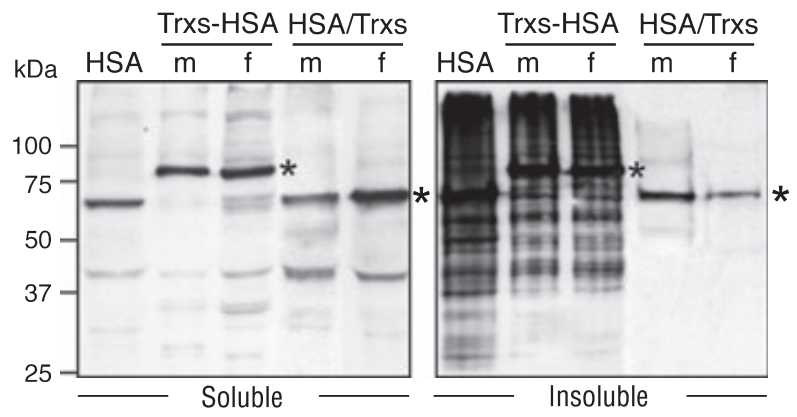

Figure 4 Analysis of soluble and insoluble fractions of transgenic lines. Ten micrograms of soluble and insoluble proteins extracted from mature leaves were loaded onto an $8 \%$ sodium dodecyl sulphate-polyacrylamide gel electrophoresis gel. Blots were detected using anti-HSA antibody. HSA: free-HSA control plant; Trxs-HSA: fusion plants; HSA/Trxs: co-expression plants. Monomers of each recombinant protein are denoted by an asterisk. Trx, thioredoxin; HSA, human serum albumin.

presence of polycistronic transcripts arising from genes located upstream was detected.

Considering the Northern blot results, post-transcriptional factors could account for high levels of HSA accumulation. Such levels could be attributed to more favourable translation or higher stability of the Trxs-fused proteins. To evaluate these factors, we performed a pulse-chase analysis of HSA protein in these plants (Figure $3 \mathrm{~b}$ ). Pulse-labelling experiments demonstrated that HSA could barely be detected after $8 \mathrm{~h}$ of chase in free-HSA expressing plants; however, in both Trxs-HSA fused lines, the recombinant protein was visible even at $48 \mathrm{~h}$ after labelling. In no case, labelled recombinant proteins were detected in untransformed control plants (Figure 3b, WT). Therefore, we concluded that the Trx-fusion strategy considerably increased the HSA half-life in chloroplasts. This result suggests that the enhancement in HSA accumulation levels is, at least to a large extent, caused by higher HSA stability inside chloroplasts.

\section{Co-expression of tobacco Trxs $\mathrm{m} / \mathrm{f}$ with HSA significantly reduced the accumulation of insoluble HSA}

In a previous report, we showed that HSA accumulated in the insoluble fraction as large aggregates or protein IBs within chloroplasts (Fernandez-San Millan et al., 2003). As we saw in Western blots (Figure 2a), Trxs-HSA fusions seem to have the same accumulation pattern as that in the free-HSA form, but a different pattern than co-expressed HSA. Because it is known that bacterial Trxs increase the solubility of fused or co-expressed proteins (Berndt et al., 2008; Kolaj et al., 2009), it might be expected that tobacco Trxs displayed a similar behaviour. Hence, soluble and insoluble protein fractions were separated from all transgenic lines and subjected to Western blot analysis (Figure 4). It was observed that soluble HSA was present in similar amounts when comparing the Trx-fusions and co-expression lines. However, Trx-fused HSA and free-HSA protein accumulated largely in the insoluble fraction. Conversely, a small amount of HSA accumulated in the insoluble fraction of Trx co-expressing lines.

Transmission electron microscopy analysis (Figures 5 and 6) was performed to determine whether the differences observed between solubility and expression among transgenic lines 


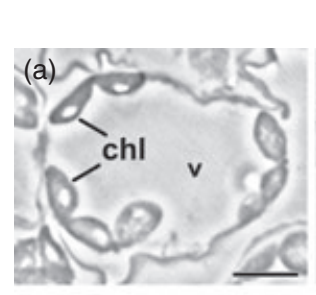

Wild type

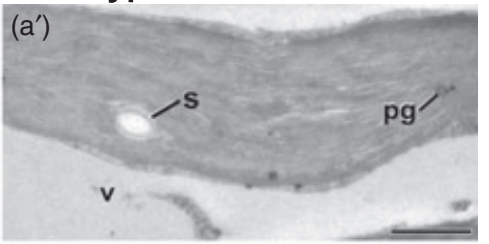

HSA

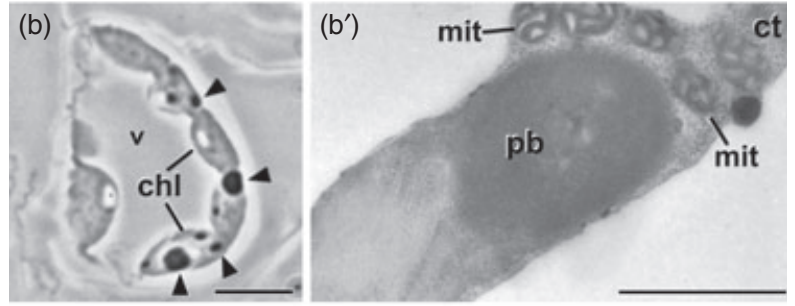

Trx-HSA fusion

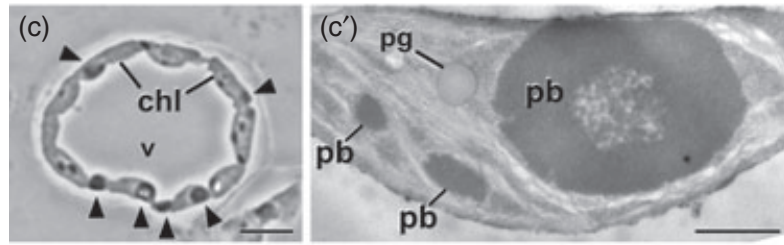

HSA/Trx co-expression

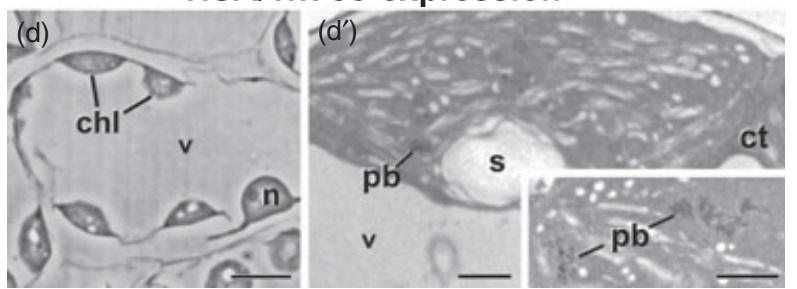

Figure $\mathbf{5}$ Ultrastructure of wild-type and transgenic HSA-expressing chloroplasts. (a-d) Architecture of leaf mesophyll cells as seen under the light microscope. $\left(a^{\prime}-d^{\prime}\right)$ Ultrastructure of chloroplasts from the corresponding cells as seen under the transmission electron microscope. $\left(a, a^{\prime}\right)$ Structure of wild-type cells (a) and chloroplasts $\left(a^{\prime}\right)$. (b, $\left.b^{\prime}\right)$ Structure of free-HSA expressing transgenic cells (b) and chloroplasts ( $\left.b^{\prime}\right)$. Note the presence of large and dense protein bodies ( $\mathrm{pb}$, arrowheads) in the chloroplasts (chl). (c, $\left.c^{\prime}\right)$ Structure of transgenic cells (c) and chloroplasts $\left(c^{\prime}\right)$ expressing a Trx-HSA fusion protein, and exhibiting even larger protein bodies. $\left(d, d^{\prime}\right)$ Structure of transgenic cells $(d)$ and chloroplasts $\left(d^{\prime}\right)$ co-expressing both HSA and Trxs. Note that the size of protein bodies in these chloroplasts is dramatically reduced and display a fibrillar texture (inset), markedly different from that of figures $b^{\prime}$ and $c^{\prime}$. ct: cytoplasm; mit: mitochondria; n: nucleus; pg: plastoglobule; s: starch; v: vacuole. Bars in a-d: $10 \mu \mathrm{m}$; $a^{\prime}-d^{\prime}: 1 \mu \mathrm{m}$. Trx, thioredoxin; HSA, human serum albumin.

correlated with structural changes within the chloroplasts. The microscopy study was performed in parallel with ultrastructural analysis and immunogold labelling. Both Trx $m$ and $f$ showed similar results under fusion and co-expression with HSA; therefore, pictures from Trx $m$ and $f$ samples are indistinctively shown. In contrast to wild-type tobacco plants (Figure 5a, $a^{\prime}$ ), transgenic chloroplasts expressing the free-HSA form presented a massive accumulation of a highly packed and electron dense material (Figure $\left.5 b, b^{\prime}\right)$, previously described as HSA protein bodies (Fernandez-San Millan et al., 2003). In plants expressing the Trxs-HSA fusion proteins, the number of these bodies
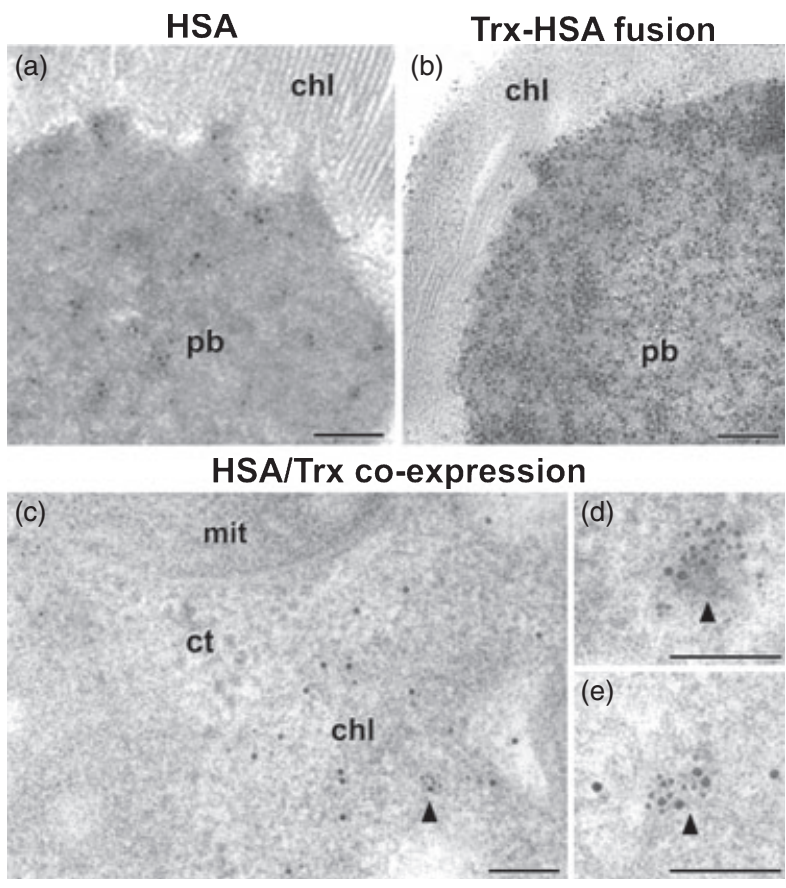

Figure 6 Immunogold labelling of transgenic HSA-expressing chloroplasts. (a) Chloroplast (chl) labelled with anti-HSA antibody, where gold particles concentrate exclusively in the protein body (pb). (b) Anti-HSA immunogold labelling of a chloroplast expressing Trx-HSA fusion. Gold particles decorate exclusively the protein bodies, independently of their size. (c-e) Double immunolocalization of HSA ( $5 \mathrm{~nm}$ gold particles) and $\operatorname{Trx}(10 \mathrm{~nm}$ gold particles) in chloroplasts co-expressing both proteins. The signal corresponding to both proteins is found exclusively within the chloroplast, where it is distributed mostly with a dispersed pattern. However, some clusters are observed (arrowheads) where particles of anti-HSA and anti-Trx can be clearly seen to co-localize ( $d$ and e). ct: cytoplasm; mit: mitochondria. Bars in a-b: 200 nm; c-e: 100 nm. Trx, thioredoxin; HSA, human serum albumin.

increased, and their sizes were larger on average (Figure $\left.5 c, c^{\prime}\right)$. However, plants co-expressing the HSA and Trxs (Figure 5d, $d^{\prime}$ ) exhibited a marked reduction in the abundance of HSA protein bodies. Most of the observed protein bodies of plants co-expressing HSA and Trxs presented a fibrillar, coil-like texture (inset within Figure $5 d^{\prime}$ ), in contrast to the solid, dense appearance of the bodies observed in the other transplastomic lines (Figure $5 b^{\prime}, c^{\prime}$ ). In addition, these bodies were markedly smaller than those observed in the chloroplasts expressing free-HSA or Trxs-HSA fusions.

Immunogold labelling with anti-HSA antibodies on free-HSA expressing chloroplasts showed labelling over the protein bodies (Figure 6a). The labelling pattern consisted of dispersed particles throughout the protein body, together with frequent clusters of 5-15 particles. In chloroplasts expressing Trxs-HSA fusions (Figure $6 b$ ), immunogold labelling over protein bodies was remarkably more abundant in both dispersed and clustered patterns. In these samples, clusters were also larger than in the free-HSA chloroplasts, comprising up to 50 particles. This finding is consistent with the higher amount of HSA quantified in these fusion plants. Outside of the protein bodies, occasional gold particles could be observed dispersed in the chloroplast stroma. No particles were identified outside the chloroplast nor in the controls that excluded the primary antibody. In chloroplasts 
co-expressing both HSA and Trxs, this scenario changed dramatically. Apart from the anti-HSA labelling found in the small and fibrillar protein bodies, most of the signal appeared dispersed throughout the stroma, with the occasional presence of clusters of about 10 gold particles (data not shown). This result suggests that co-expression of HSA and Trxs somehow affects the distribution pattern of HSA.

A co-localization experiment of Trxs and HSA was performed to shed light on potential interactions between both proteins in HSA co-expressing chloroplasts. As revealed by anti-HSA and anti-Trxs double immunogold labelling ( 5 and $10 \mathrm{~nm}$ gold particles, respectively; Figure $6 \mathrm{c}$ ), HSA-labelled proteins followed the previously described patterns of distribution: gold particles were mostly dispersed with some occasional clusters. The anti-Trx particles presented a similarly dispersed pattern. Interestingly, in the anti-HSA clusters, the presence of anti-Trx particles was frequently detected as being either included within the cluster or at a distance close enough to be considered co-localizing with the anti-HSA particles (Figure $6 \mathrm{~d}$ ). Some of the dual clusters of anti-HSA and anti-Trx particles were observed to decorate dense stromal structures, very similar to small, growing protein bodies (Figure 6e).

\section{Tobacco thioredoxins $\mathrm{m}$ and $\mathrm{f}$ show holdase activity}

Holdase chaperones are proteins that bind to folding intermediates, thereby preventing their nonspecific aggregation (Beissinger and Buchner, 1998). Because it is known that holdase function is a peculiar characteristic of some plant Trxs (Lee et al., 2009; Park et al., 2009), we wanted to study whether the tobacco plastidial Trxs could act as holdase chaperones, which could account to some extent for the absence of large IBs in HSA co-expressing lines. Trx $m$ and $f$ were bacterially expressed and purified to homogeneity. Holdase chaperone activity was analysed by measuring the ability of each Trx to inhibit the thermal aggregation of malate dehydrogenase $(\mathrm{MDH})$, which has often been employed as a model polypeptide substrate to confirm the holdase function of other plant Trxs (Lee et al., 2009; Park et al., 2009). Incubation of $2 \mu \mathrm{M} \mathrm{MDH}$ with increasing amounts of Trxs (2-12 $\mu \mathrm{m})$ gradually prevented the thermal aggregation of $\mathrm{MDH}$ (Figure 7). This aggregation was significantly blocked at a $6-\mu \mathrm{M}$ concentration of Trx m, which is equivalent to a subunit molar ratio of $1 \mathrm{MDH}$ to $3 \mathrm{Trx}$ $\mathrm{m}$. It appeared that Trx $\mathrm{m}$ prevented $\mathrm{MDH}$ aggregation more efficiently than Trx f; however, both Trxs act as molecular holdases at high concentrations $(12 \mu \mathrm{M})$. When Trxs were replaced with bovine serum albumin (BSA), holdase chaperone activity was not detected, suggesting that this activity was derived from the specific function of Trxs. A direct inhibition of HSA thermal aggregation in vitro was also attempted, but its physical properties do not allow us to perform this assay as HSA is even more stable at high temperatures than the assayed Trxs.

\section{Discussion}

In a previous work (Fernandez-San Millan et al., 2003, 2007), it was demonstrated that HSA, a complex globular protein with 17 disulphide bonds and highly susceptibility to degradation in chloroplasts, accumulated almost completely as protein IBs. It is widely known that protein aggregation into IBs mostly involves intermolecular associations of partially folded intermediates (Mitraki and King, 1989). Therefore, it can be deduced that in this case, HSA most likely did not have a native structure within

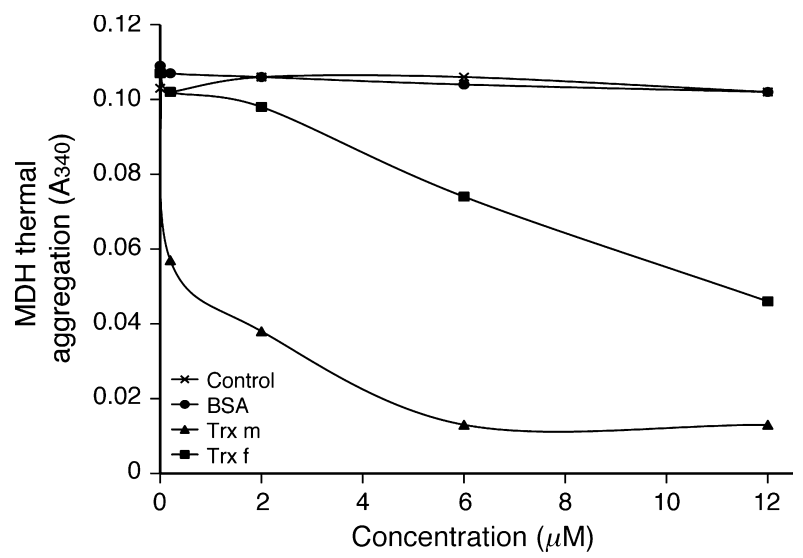

Figure 7 Holdase chaperone activity of tobacco $\operatorname{Trx} \mathrm{m} / \mathrm{f}$. Thermal aggregation at $43^{\circ} \mathrm{C}$ of $2 \mu \mathrm{m}$ of malate dehydrogenase (MDH) was examined for $60 \mathrm{~min}$ in the absence $(x)$ or presence of different concentrations of $\operatorname{Trx} m(\mathbf{\Delta})$ or $f(\boldsymbol{\square})$, by monitoring the turbidity increase at $340 \mathrm{~nm}$. Reactions were also measured using same concentrations of bovine serum albumin $(\bullet)$ instead of Trxs. Representative plot of three independent experiments is shown. Trx, thioredoxin.

plastids, although chloroplasts are able to accomplish the correct formation of disulphide bridges and fold foreign proteins in a biologically active form (Staub et al., 2000; Daniell et al., 2001; Molina et al., 2004; Ruhlman et al., 2007; Farran et al., 2008). Production of recombinant protein as insoluble aggregates can offer the advantage of easy purification (Yasukawa et al., 1995; Fernandez-San Millan et al., 2003); however, in vitro manipulations must be made to refold the heterologous protein, and further, the native protein conformation is not guaranteed (Yasukawa et al., 1995). Thus, large-scale production of proteins in biologically active soluble form is highly desirable as it facilitates downstream processing and lowers total costs. Fusion or co-expression techniques with proteins such as Trxs are common approaches in microbial systems for the expression of small peptides and unstable or insoluble proteins that accumulate at low levels or in the insoluble fraction (Berndt et al., 2008; Demain and Vaishnav, 2009; Kolaj et al., 2009). Thus, the lack of stability and solubility of HSA expressed in tobacco chloroplasts prompted us to investigate whether we could improve its intraplastidic expression using an $\mathrm{N}$-terminal protein fusion or co-expression strategy. Because of their endosymbiotic origin, chloroplasts harbour genetic, structural and metabolic resemblances with prokaryotic cells (McFadden, 2001). For instance, plant chloroplast Trx $m$ has a prokaryotic origin and strongly resembles bacterial Trxs (Meyer et al., 2005). Thus, it seems conceivable that the transference of bacterial Trxs properties to the chloroplast expression system is possible.

In this context, we have assessed the well-known plastids Trxs $m$ and $f$ as heterologous partners for HSA expression in chloroplasts. In this model system, we hypothesized that the folding and accumulation of HSA in transgenic plastids might also benefit from the biological activities of chloroplast Trxs. To test this hypothesis, we generated four different chloroplast transformation vectors for HSA fusion and co-expression with either Trx $m$ or $f$. Our results demonstrate an improvement in HSA accumulation mediated by Trx $m$ or $f$ fusions. HSA accumulated between $26 \%$ and $22 \%$ of the TP, which represents, 
on average, a 3.5-fold increase in the expression level achieved in control free-HSA expressing plants (Figure 2e). Expression improvement of other recombinant proteins fused to these tobacco Trxs has been corroborated in our laboratory (unpublished data). In contrast, the presence of Trxs in co-expressing HSA plants resulted in an HSA decrease up to 4.5 -fold. Such variability in HSA protein accumulation in transplastomic plants did not appear to depend on differences at the mRNA level (Figure 3a). Although Trxs-fusion plants accumulated the highest levels of HSA, slightly less amount of HSA transcripts were detected in fusions or co-expressions when compared to freeHSA control plants. Trx transcripts were also analysed by Northern blot, which showed monocistrons for the fusion lines and mono- and dicistrons for the co-expressing lines. However, even though $\operatorname{Tr} x$ monocistrons were significantly more abundant in co-expressing than in fusion plants, this difference did not translate into higher levels of Trxs accumulation, as was observed by protein quantification (Figure 2e). Hence, we can conclude that other post-transcriptional factors must be influencing HSA and Trxs accumulation within chloroplasts.

It is known that plastid protein expression is extensively controlled at the post-translational level, mainly via regulated translation, protein complex assembly and proteolysis (Koop et al., 2007). A more favourable translation or higher degree of protein stability could account for the increase in HSA accumulation levels in the fusion plants. Experiments in plastid expression of recombinant proteins have shown that a crucial factor in obtaining high levels of gene expression is the efficiency of translation initiation (Kuroda and Maliga, 2001b), which is very sensitive to the nucleotide sequence surrounding the initiating methionine codon. For this reason, modifications or extensions of the $\mathrm{N}$-terminus of the desired protein often facilitate protein accumulation (Ye et al., 2001; Herz et al., 2005; Lenzi et al., 2008; Scotti et al., 2009; Farran et al., 2010). However, Trxs could confer stability to the molecule by protecting HSA from proteolytic degradation. As mentioned previously, it is known that stable proteins (e.g. GFP or GUS) in fusion with less stable partners confer a certain degree of stability (Leelavathi and Reddy, 2003; Molina et al., 2004; Lee et al., 2010; Lentz et al., 2010). Pulse-chase analysis of Trxs-HSA fusion proteins demonstrated a marked increase in stability (Figure 3b). We can conclude that higher accumulation levels of the Trxs-HSA fusions are mainly caused by their high stability inside chloroplasts. Similarly, Leelavathi and Reddy (2003) demonstrated a direct relation between stability and recombinant protein yield when IFN-g was expressed alone or translationally fused to the GUS protein.

Escherichia coli TrxA fusion and co-expression is a strategy to increase not only levels of expression but also solubility in microbial systems (Berndt et al., 2008; Kolaj et al., 2009). Knowing these Trx capabilities, the distribution of HSA in soluble and insoluble fractions of plants expressing Trxs-HSA fusions and co-expressions in chloroplasts was analysed and compared with that of free-HSA expressing control line. As demonstrated previously (Fernandez-San Millan et al., 2003), free-HSA was recovered mainly in the insoluble fraction and was detected at very low levels in the soluble fraction, indicating the presence of protein aggregates. The same pattern was observed in the Trxs-HSA fusions. Nevertheless, although similar amounts of HSA were found in soluble fractions of co-expressing plants, low quantities of HSA were found in the insoluble fractions (Figure 4). To further investigate this observation, transmission electron microscopy and immunogold labelling were performed (Figures 5 and 6). As expected, similar to free-HSA expressing control plants, electron micrographs showed the formation of large protein IBs within chloroplasts expressing the Trxs-HSA fusion proteins. The number and size of these bodies increased in fusion plants compared to free-HSA control plants. Furthermore, HSA abundance also increased in protein bodies, as revealed by anti-HSA immunogold labelling, likely as a consequence of an enhancement in the protein stability mediated by Trxs as mentioned earlier. These results indicate that linkage of HSA to Trx $m$ and $f$ failed to increase HSA solubility in chloroplasts. This finding is in contrast to what has been previously observed for a number of insoluble proteins fused to E. coli TrxA in bacterial expression systems. However, in some cases, bacterial Trx fusions are not able to inhibit recombinant protein IB accumulation, although the causes remain unclear (Hammarstrom et al., 2002). Dense aggregates practically disappeared when HSA was co-expressed with either Trx $m$ or $f$. In these plants, electron micrographs showed small protein bodies with a fibrillar texture, suggesting a possible influence of Trxs on HSA solubility. Utilizing the approach previously used (Anderson et al., 2008), we showed that HSA co-localizes with Trxs (Figure $6 \mathrm{c}-\mathrm{e})$. Association and interaction in vivo is difficult or impossible to prove conclusively; however, our co-localization experiment indicates that Trxs are not randomly distributed with respect to HSA. Thus, it is likely that HSA associates with Trxs in situ. In conclusion, it seems that Trx expression in a free or a fused form has different effects on HSA solubility, which would explain the protein IB formation only when HSA is fused to Trx. Different effects of fused or co-expressed E. coli TrxA over recombinant proteins have been reported previously (Yuan et al., 2004).

Considering the association observed between Trxs and HSA in co-localization experiments and that chaperones interact preferentially with unfolded proteins (Hartl, 1996), we decided to confirm the putative chaperone activity of tobacco Trxs $m$ and $f$ used in this study. Chaperones have been shown to partially promote the folding of proteins by preventing their aggregation (holdase activity) (Beissinger and Buchner, 1998). This activity has recently been demonstrated in vitro for two Arabidopsis thaliana proteins: a Trx h (Park et al., 2009) and a Trx-like protein (Lee et al., 2009). In this study, the holdase chaperone activity of Trx $m$ and $f$ was analysed (Figure 7). Although a direct effect of plastid Trxs on HSA aggregation has not been experimentally demonstrated in vitro, our results provide biochemical evidence to confirm that tobacco Trx $m$ and $f$ have a holdase-like activity, as both Trxs are able to prevent MDH thermal aggregation. Based on these observations, the hypothetical Trx $m$ and $f$ holdase activity in vivo might also explain why the protein bodies practically disappear in chloroplasts of co-expressing lines. In this case, Trxs might increase the solubility by preventing aggregation and precipitation of nascent proteins, giving them an extended opportunity to adopt their correct tertiary folding. We also hypothesize that the lack of large HSA aggregates in these co-expression lines makes HSA more accessible to proteases, thus explaining the low levels of HSA.

\section{Concluding remarks}

Four conclusions can be drawn from this work: (i) linkage of Trxs and HSA in chloroplasts significantly increases the final yield of accumulated HSA; (ii) N-terminal Trx fusions confer high 
stability to HSA; (iii) co-expression of tobacco Trxs with HSA in chloroplasts restricts large IB formation, although these plants do not accumulate more soluble HSA than control plants; and (iv) a similar behaviour of $\operatorname{Trx} m$ and $f$ as modulators of HSA production in chloroplasts is observed despite the fact that their functions and phylogenetic origins have been reported to be different.

Thus, although there is a continuing need for new and easier methods to produce recombinant proteins for use in research, diagnostic and therapeutic applications, there are many problems such as low levels of expression, instability, insolubility and inappropriate folding that need to be addressed. At present, there are some alternatives for increasing the level of expression or stability, but to date, no research has focused on minimizing aggregate formation or improving folding of recombinant proteins produced by the transplastomic system. In this study, we showed that tobacco Trx-fusion or co-expression with HSA is a useful and promising strategy. Our results provide evidence that Trxs might exert an important role in modulating solubility patterns and improving the recombinant protein stability and final yield. To understand in-depth the potential of Trx proteins, other studies are ongoing with structurally simpler proteins than HSA and with a biological function that is easier to measure.

\section{Experimental procedures}

\section{Construction of the chloroplast expression vectors}

Nicotiana tabacum Trx $m$ and $f$ sequences (GenBank accession HQ338525 and HQ338526 respectively) were used for the design of primers to amplify the mature coding region from tobacco CDNA, previously obtained by RT-PCR. For the fusion strategy, Trx $m$ and $f$ were amplified by PCR with the following primers to introduce an $\mathrm{NCOl}$ site at the $5^{\prime}$ end and a Smal site at the $3^{\prime}$ end, and to eliminate the TAA termination codon: FusTrxm-For 5'-CCATGGGTGAAGCGCAAA-3' and FusTrxm-Rev 5'-CCCGGGCAAGAATTTCTCTATGCAGGTGG-3'; FusTrxf-For 5'-CCATGGGTAGCTCCGATGCTACTG-3' and FusTrxf-Rev 5'-GCGGCCGCACTTGACCGCACATCCTCAATTG-3'. PCR products were cloned into the pGEM-T vector (Promega, Madison, WI). HSA was cloned with Smal and Notl sites at the $5^{\prime}$ and $3^{\prime}$ ends, respectively, by PCR from the pLD-psbAHSA vector (Fernandez-San Millan et al., 2003) with the following primers: HSA-For 5'-GGGCCAGGGCCAATGAGCCGGAGG-3' and HSARev 5'-GCGGCCGCTTAGGCCGAGCCCCC-3'. Trx and HSA sequences were fused together and to the promoter and $5^{\prime}$-UTR of the tobacco psbA gene in a pKS intermediate vector (Promega). Finally, the fusion expression cassette was digested by ECoRV and Notl and introduced into the pLD vector (Daniell et al., 1998; Fernandez-San Millan et al., 2003). The resulting vectors were named pLD-FusTrxm-HSA and pLD-FusTrxf-HSA.

For the co-expression strategy, $\operatorname{Trx} m$ and $f$ were amplified by PCR with the following primers to introduce the Scal and Notl sites at the $5^{\prime}$ and $3^{\prime}$ ends, respectively: CoTrxm-For 5'-AGTACTTGAAGCGCAAA-3' and CoTrxm-Rev 5'-GCGGCCGCTTAC AAGAATTTCTCTATGCAGGTGG-3'; CoTrxf-For 5'-AGTACTTAGCTCCGATGCTACTG-3' and CoTrxf-Rev 5'-GCGGCCGCTTAACTTGACCGCACATCCTCAATTG-3' and cloned into the pGEM-T vector (Promega). In this vector, the PrrnG10L promoter and leader region (Farran et al., 2008) were fused to $\operatorname{Tr} x$ $m / f$ sequences at the $5^{\prime}$ end. The product PrrnG10L-Trx $m / f$ was excised with Notl and introduced into the pLD-psbAHSA vector (Fernandez-San Millan et al., 2003) downstream of the HSA gene. The resulting vectors were named pLD-CoHSA-Trxm and pLD-COHSA-Trxf.

The final clones were sequenced, and their functionality was tested by Western blot of E. coli culture extracts.

\section{Expression and purification of tobacco Trx $\mathrm{m} / \mathrm{f}$ from Escherichia coli and production of polyclonal antibodies}

Tobacco Trx $m$ and Trx $f$ were expressed in E. coli as His-tagged polypeptides. The coding sequences were amplified by PCR with the same primers as those used for their cloning (FusTrxm/f-For and CoTrxm/f-Rev); the resulting PCR fragment was digested with $\mathrm{Ncol}$ and Notl and cloned in a pET 28a(+) vector (Novagene; Merck KGaA, Darmstadt, Germany). The resulting plasmids, termed pET Trxm and $\mathrm{pET}$ Trxf, were introduced into E. coli (BLR DE3, Novagene). Overexpressed proteins were purified by chromatography in affinity columns packed with Ni-NTA agarose (Qiagen, Hilden, Germany). Specific anti-Trx $m$ and anti-Trx $f$ antibodies were raised by immunizing rabbits with purified His-tagged proteins (Abyntek, Bilbao, Spain). Antibody cross-reactivity was eliminated by affinity purification (exclusion) of anti-Trx $m$ lgG with the Trx $f$ antigen and vice versa.

\section{Bombardment and regeneration of chloroplast transgenic plants}

Gold microprojectiles $(0.6 \mu \mathrm{m})$ coated with plasmid DNA (pLDFusTrxm-HSA, pLD-FusTrxf-HSA; pLD-COHSA-Trxm and pLD-CoHSA-Trxf) were bombarded into in vitro-grown tobacco leaves (Nicotiana tabacum cv. Petite Havana SR1; National Germplasm Resources Laboratory, Beltsville, MD) using the biolistic device PDS1000/He (Bio-Rad, Hercules, CA) as previously described (Daniell, 1997). After bombardment of the abaxial leaf side, leaves were cut into small pieces (c. $5 \times 5 \mathrm{~mm}$ ) and placed adaxial side up on Magenta vessels (Sigma-Aldrich, St Louis, $\mathrm{MO}$ ) containing $500 \mathrm{mg} / \mathrm{L}$ spectinomycin dihydrochloride as a selecting agent. Resistant shoots were subjected to a second round of selection under the same conditions. PCR was used to analyse integration of different cassettes in the transformed plants with the following primers: primer 3P $5^{\prime}$-AAAACCCGTCCTCAGTTCGGATTGC-3', which binds on the chloroplast genome upstream of the $t r n /$ gene outside the vector integration site, and primer 3M 5'-CCGCGTTGTTTCATCAAGCCTTACG-3', which binds to the aadA gene.

\section{Southern and Northern blot analyses}

Total plant DNA was extracted from leaves using the cetyltrimethylammonium bromide procedure. Ten micrograms of total DNA were digested with HindIII, separated on a $0.8 \%(\mathrm{w} / \mathrm{v})$ agarose gel and transferred to a nylon membrane. The digestion by $\mathrm{Bg} / \mathrm{ll}$ and $\mathrm{BamHI}$ of the pFS vector generated a $0.8-\mathrm{kb}$ probe (FS) homologous to the flanking sequences. A $0.75-\mathrm{kb}$ HSA probe was obtained by Ncol-Notl digestion of pLD-psbAHSA vector (Fernandez-San Millan et al., 2003). A 0.37-kb fragment (Trxm probe) of the Trx $m$ gene and a $0.39-\mathrm{kb}$ fragment (Trxf probe) of the Trx $f$ gene were generated by PCR with the same primers used for their cloning. Hybridization was performed using the chemiluminescent AlkPhos direct labellingdetection system (GE Healthcare, Buckinghamshire, UK). After Southern blot confirmation, plants were transferred to soil. Seeds from the $T_{0}$ generation were germinated in vitro on the 
spectinomycin selection medium. The $T_{1}$ seedlings were isolated and cultured for 4 weeks in Magenta vessels. Finally, plants were transferred to pots. Plants from the $T_{0}$ and $T_{1}$ generations were analysed for homoplasmy.

Total RNA was extracted (Ultraspec RNA; Biotecx Laboratories, Houston, TX) from leaves of transformed and untransformed plants. RNA $(10 \mu \mathrm{g})$ was electrophoresed on $1.5 \%$ agarose/formaldehyde gels and then transferred to a nylon membrane. The previously described HSA- and Trx-specific probes were used. Labelling and hybridization were performed using the chemiluminescent detection system mentioned earlier. Ethidium bromide-stained total leaf RNA was used to assess loading.

\section{Western blot analysis}

Transformed and untransformed leaves of plants grown in a greenhouse were ground in liquid nitrogen. Leaves $(100 \mathrm{mg})$ were homogenized in $600 \mathrm{~mL}$ of Laemmli buffer $(0.5 \mathrm{M}$ Tris- $\mathrm{HCl}$ $\mathrm{pH} 6.5,4 \%$ SDS, $20 \%$ glycerol and 10\% $\beta$-mercaptoethanol) and heated at $95{ }^{\circ} \mathrm{C}$ for $5 \mathrm{~min}$. After $5 \mathrm{~min}$ of centrifugation at $20000 \mathbf{g}$, the supernatant was considered the TP. TP was measured using the RC-DC protein assay (Bio-Rad) with BSA as a standard, according to the manufacturer's instructions. For soluble extractions, $100 \mathrm{mg}$ of leaves were homogenized in $600 \mu \mathrm{L}$ of buffer (Ouerghi et al., 2000). After centrifugation at $20000 \mathbf{g}$ for $5 \mathrm{~min}$, the supernatant was considered the soluble fraction. The pellet was re-suspended in $200 \mu \mathrm{L}$ of Laemmli buffer and boiled for $5 \mathrm{~min}$. After centrifugation at $20000 \mathrm{~g}$ for $5 \mathrm{~min}$, the supernatant was considered the insoluble fraction. Soluble and insoluble proteins were measured using the Bradford or RC-DC protein assay (Bio-Rad), respectively. Proteins were separated by SDS-PAGE on $8 \%-13 \%$ polyacrylamide gels and transferred to a nitrocellulose membrane for immunoblotting. Anti-HSA (Nordic Immunology, Tilburg, the Netherlands) at 1:10 000 dilution or anti-Trx $\mathrm{m} / \mathrm{f}$ antibodies (Abyntek) at $1: 5000 / 1: 7500$ dilution were used as primary antibodies. Peroxidase-conjugated goat anti-rabbit immunoglobulin G (Sigma-Aldrich) was used as the secondary antibody at $1: 10000$ dilution. Detection was performed using the chemiluminescence ECL Western blotting system (GE Healthcare).

Quantification of HSA and Trxs was performed by comparing dilution series of TP from transgenic plants with dilution series of purified HSA (Sigma-Aldrich) or Trxs (purified from E. coli). For each protein, adequate amounts were loaded on an SDSPAGE gel, electrophoretically separated and then analysed by Western blot. Immunoblots were quantified using the GeneTools analyzer software (SynGene, Cambridge, UK).

\section{Pulse-chase analysis}

Leaf discs $\left(0.97 \mathrm{~cm}^{2}\right)$ from young, fully expanded leaves of transformed and nontransformed plants growing in a greenhouse were sampled, layered immediately on $0.5 \mathrm{~mL}$ of Murashige and Skoog (Duchefa, Haarlem, the Netherlands) liquid medium, $\mathrm{pH} 5.8$ containing $40 \mu \mathrm{Ci}$ of a ${ }^{35} \mathrm{~S}$-methionine and ${ }^{35}$-S-cysteine (Perkin Elmer, Boston, MA) mixture per leaf disc and vacuum infiltrated for $3 \mathrm{~min}$. Leaf discs were then illuminated for $1 \mathrm{~h}$ at $25^{\circ} \mathrm{C}$, blotted briefly with blotting paper, rinsed by vacuum infiltration with $2.5 \mathrm{~mL}$ of water per disc, blotted again and vacuum infiltrated with Murashige and Skoog liquid medium containing cold $10 \mathrm{~mm}$ methionine and $5 \mathrm{~mm}$ cysteine. Samples were stored in a culture chamber under continuous light for the different chase periods (up to $48 \mathrm{~h}$ ). Three leaf discs were harvested and frozen in liquid nitrogen per time-point. Leaf samples were ground in $150 \mu \mathrm{L}$ of Laemmli buffer and boiled for $5 \mathrm{~min}$. Protein samples were separated by $8 \%$ SDS-PAGE. The resulting gels were dried and exposed to film for 4 days.

\section{Light and electron microscopy and immunogold labelling}

Leaf samples from plants grown in a growth chamber were fixed in Karnovsky fixative (4\% formaldehyde and $5 \%$ glutaraldehyde in $0.025 \mathrm{M}$ cacodylate buffer, $\mathrm{pH} 6.7$ ), post-fixed in $2 \% \mathrm{OsO}_{4}$, dehydrated in methanol series for 3 days and slowly embedded in Epon resin for 2 days. Epon blocks were polymerized at $60^{\circ} \mathrm{C}$ for 2 days. For light microscopy, semi-thin $(1 \mu \mathrm{m})$ sections were obtained, stained with toluidine blue, mounted and observed in a Nikon Eclipse E1000 microscope. For ultrastructural observations, thin sections (c. $80 \mathrm{~nm}$ ) were collected on 100-mesh copper grids, counterstained with uranyl acetate and lead citrate, and observed using a Philips CM10 transmission electron microscope (Philips, Hillsboro, OR) operating at $100 \mathrm{kV}$.

For single immunogold labelling, leaf samples were fixed in $4 \%$ formaldehyde in phosphate buffer saline (PBS) and dehydrated in methanol by the 'Progressive Lowering of Temperature' (PLT) method in a Leica AFS automated system (Leica, Wetzlar, Germany). Then, samples were infiltrated in Lowicryl $\mathrm{K} 4 \mathrm{M}$ resin and polymerized at $-30^{\circ} \mathrm{C}$ under ultraviolet light. For immunolabelling, Lowicryl sections (thickness, c. $80 \mathrm{~nm}$ ) were deposited on Formvar- and carbon-coated nickel grids. Immunogold labelling was performed as described previously (Segui-Simarro et al., 2003). Briefly, sections were hydrated, floated in PBS, blocked with 5\% BSA in PBS and incubated with goat anti-HSA (Bethyl Laboratories Inc., Montgomery, TX) or rabbit anti-Trx $\mathrm{m} / \mathrm{f}$ (Abyntek) antibodies diluted $1: 2$ and $1: 5$, respectively, in $1 \%$ BSA for $1 \mathrm{~h}$ at room temperature. Sections were then incubated with an anti-goat lgG antibody for the anti-HSA detection, or with an anti-rabbit IgG antibody for the anti-Trxs detection, both from British Biocell International (Cardiff, UK) and conjugated to $10-\mathrm{nm}$ gold particles, diluted $1: 25$ in $1 \%$ BSA for 45 min. Finally, sections were washed, air dried, counterstained with uranyl acetate and lead citrate and observed in a Philips CM10 transmission electron microscope operating at $100 \mathrm{kV}$. Controls were performed excluding the corresponding primary antibody from the incubation buffer.

For co-localization experiments, double immunogold labelling was performed essentially following the protocol described earlier, but a mixture of anti-HSA and anti-Trx $m$ or $f$ primary antibodies diluted as previously mentioned was used instead. Then, sections were incubated with a mixture of an anti-goat lgG antibody conjugated to 5-nm gold particles (British Biocell International) for the specific detection of the anti-HSA antibody and with the previously described anti-rabbit IgG antibody conjugated to 10-nm gold particles for the detection of the anti-Trx $\mathrm{m} / \mathrm{f}$ antibodies, both diluted $1: 25$ in 1\% BSA for $45 \mathrm{~min}$. Controls were performed by excluding both primary antibodies from the incubation buffer.

\section{Holdase activity assay}

Thermal aggregation of MDH was used as holdase activity assay (Park et al., 2009). MDH from porcine heart (Sigma-Aldrich) was incubated at $2 \mu \mathrm{m}$ in $50 \mathrm{~mm}$ HEPES-KOH (pH 8.0) buffer at 
$43{ }^{\circ} \mathrm{C}$ with various concentrations of $\operatorname{Trxs}(1: 1,3: 1$ and $6: 1$ Trx: $\mathrm{MDH}$ molar ratio). Thermal aggregation of $\mathrm{MDH}$ was determined in a microtiter plate by monitoring the turbidity increase at $340 \mathrm{~nm}$ in a temperature-controlled spectrophotometer (Spectra Max 340PC; Molecular Devices, Sunnyvale, CA). BSA was used as control.

\section{Acknowledgements}

Authors wish to thank MJ Villafranca for excellent plant care and cultivation. This work was supported by grants Res.17/2004 and IIM10865.RI1 (Proyecto EUROINNOVA) from Gobierno de Navarra (Spain). RSB and PCM were supported by predoctoral fellowships from CSIC and Generalitat Valenciana, respectively. AFS was supported by a postdoctoral fellowship from Public University of Navarra.

\section{References}

Anderson, L.E., Fadowole, D., Reyes, B.A. and Carol, A.A. (2008) Distribution of thioredoxins $f$ and $m$ with respect to seven light-activated enzymes and three redox-insensitive proteins in pea leaf chloroplasts. Plant Sci. 174, 432-445.

Arsova, B., Hoja, U., Wimmelbacher, M., Greiner, E., Ustun, S., Melzer, M., Petersen, K., Lein, W. and Bornke, F. (2010) Plastidial thioredoxin Z interacts with two fructokinase-like proteins in a thiol-dependent manner: evidence for an essential role in chloroplast development in Arabidopsis and Nicotiana benthamiana. Plant Cell, 22, 1498-1515.

Beissinger, M. and Buchner, J. (1998) How chaperones fold proteins. Biol. Chem. 379, 245-259.

Berndt, C., Lillig, C.H. and Holmgren, A. (2008) Thioredoxins and glutaredoxins as facilitators of protein folding. Biochim. Biophys. Acta, 1783, 641-650.

Bessette, P.H., Aslund, F., Beckwith, J. and Georgiou, G. (1999) Efficient folding of proteins with multiple disulfide bonds in the Escherichia coli cytoplasm. Proc. Natl. Acad. Sci. U S A, 96, 13703-13708.

Bogomolovas, J., Simon, B., Sattler, M. and Stier, G. (2009) Screening of fusion partners for high yield expression and purification of bioactive viscotoxins. Protein Expr. Purif. 64, 16-23.

Buchanan, B.B. and Balmer, Y. (2005) Redox regulation: a broadening horizon. Annu. Rev. Plant. Biol. 56, 187-220.

Daniell, H. (1997) Transformation and foreign gene expression in plants by microprojectile bombardment. Methods Mol. Biol. 62, 463-489.

Daniell, H., Datta, R., Varma, S., Gray, S. and Lee, S.B. (1998) Containment of herbicide resistance through genetic engineering of the chloroplast genome. Nat. Biotechnol. 16, 345-348.

Daniell, H., Lee, S.B., Panchal, T. and Wiebe, P.O. (2001) Expression of the native cholera toxin $B$ subunit gene and assembly as functional oligomers in transgenic tobacco chloroplasts. J. Mol. Biol. 311, 1001-1009.

Daniell, H., Ruiz, G., Denes, B., Sandberg, L. and Langridge, W. (2009) Optimization of codon composition and regulatory elements for expression of human insulin like growth factor-1 in transgenic chloroplasts and evaluation of structural identity and function. BMC Biotechnol. 9, 33.

Demain, A.L. and Vaishnav, P. (2009) Production of recombinant proteins by microbes and higher organisms. Biotechnol. Adv. 27, 297-306.

Farran, I., Rio-Manterola, F., Iniguez, M., Garate, S., Prieto, J. and MingoCastel, A.M. (2008) High-density seedling expression system for the production of bioactive human cardiotrophin-1, a potential therapeutic cytokine, in transgenic tobacco chloroplasts. Plant Biotechnol. J. 6, 516-527.

Farran, I., McCarthy-Suarez, I., Rio-Manterola, F., Mansilla, C., Lasarte, J.J. and Mingo-Castel, A.M. (2010) The vaccine adjuvant extra domain A from fibronectin retains its proinflammatory properties when expressed in tobacco chloroplasts. Planta, 231, 977-990.

Fernandez-San Millan, A., Mingo-Castel, A., Miller, M. and Daniell, H. (2003) A chloroplast transgenic approach to hyper-express and purify human serum albumin, a protein highly susceptible to proteolytic degradation. Plant Biotechnol. J. 1, 71-79.
Fernandez-San Millan, A., Farran, I., Molina, A., Mingo-Castel, A.M. and Veramendi, J. (2007) Expression of recombinant proteins lacking methionine as $\mathrm{N}$-terminal amino acid in plastids: human serum albumin as a case study. J. Biotechnol. 127, 593-604.

Fernandez-San Millan, A., Ortigosa, S.M., Hervas-Stubbs, S., Corral-Martinez, P., Segui-Simarro, J.M., Gaetan, J., Coursaget, P. and Veramendi, J. (2008) Human papillomavirus L1 protein expressed in tobacco chloroplasts selfassembles into virus-like particles that are highly immunogenic. Plant Biotechnol. J. 6, 427-441.

Garcia-Ortega, L., Lacadena, J., Lacadena, V., Masip, M., De Antonio, C., Martinez-Ruiz, A. and Martinez Del Pozo, A. (2000) The solubility of the ribotoxin alpha-sarcin, produced as a recombinant protein in Escherichia coli, is increased in the presence of thioredoxin. Lett. Appl. Microbiol. 30, 298-302.

Hammarstrom, M., Hellgren, N., van Den Berg, S., Berglund, H. and Hard, T. (2002) Rapid screening for improved solubility of small human proteins produced as fusion proteins in Escherichia coli. Protein Sci. 11, 313-321.

Hartl, F.U. (1996) Molecular chaperones in cellular protein folding. Nature, 381, 571-579.

Herz, S., Fussl, M., Steiger, S. and Koop, H.U. (2005) Development of novel types of plastid transformation vectors and evaluation of factors controlling expression. Transgenic Res. 14, 969-982.

lamtham, S. and Day, A. (2000) Removal of antibiotic resistance genes from transgenic tobacco plastids. Nat. Biotechnol. 18, 1172-1176.

Issakidis-Bourguet, E., Mouaheb, N., Meyer, Y. and Miginiac-Maslow, M. (2001) Heterologous complementation of yeast reveals a new putative function for chloroplast m-type thioredoxin. Plant J. 25, 127-135.

Jurado, P., de Lorenzo, V. and Fernandez, L.A. (2006) Thioredoxin fusions increase folding of single chain Fv antibodies in the cytoplasm of Escherichia coli: evidence that chaperone activity is the prime effect of thioredoxin. J. Mol. Biol. 357, 49-61.

Kolaj, O., Spada, S., Robin, S. and Wall, J.G. (2009) Use of folding modulators to improve heterologous protein production in Escherichia coli. Microb. Cell. Fact. 8, 9.

Koop, H.U., Herz, S., Golds, T.J. and Nickelsen, J. (2007) The genetic transformation of plastids. In Cell and Molecular Biology of Plastids, Vol. 19 (Bock, R., ed.), pp. 457-510, Berlin, Heidelberg: Springer-Verlag.

Kumar, J.K., Tabor, S. and Richardson, C.C. (2004) Proteomic analysis of thioredoxin-targeted proteins in Escherichia coli. Proc. Natl. Acad. Sci. U S A, 101, 3759-3764.

Kuroda, H. and Maliga, P. (2001a) Sequences downstream of the translation initiation codon are important determinants of translation efficiency in chloroplasts. Plant Physiol. 125, 430-436.

Kuroda, H. and Maliga, P. (2001b) Complementarity of the 165 rRNA penultimate stem with sequences downstream of the AUG destabilizes the plastid mRNAs. Nucleic Acids Res. 29, 970-975.

LaVallie, E.R. and McCoy, J.M. (1995) Gene fusion expression systems in Escherichia coli. Curr. Opin. Biotechnol. 6, 501-506.

LaVallie, E.R., Rehemtulla, A., Racie, L.A., DiBlasio, E.A., Ferenz, C., Grant, K.L., Light, A. and McCoy, J.M. (1993) Cloning and functional expression of a CDNA encoding the catalytic subunit of bovine enterokinase. J. Biol. Chem. 268, 23311-23317.

LaVallie, E.R., Lu, Z., Diblasio-Smith, E.A., Collins-Racie, L.A. and McCoy, J.M. (2000) Thioredoxin as a fusion partner for production of soluble recombinant proteins in Escherichia coli. Methods Enzymol. 326, 322340.

LaVallie, E.R., DiBlasio-Smith, E.A., Collins-Racie, L.A., Lu, Z. and McCoy, J.M. (2003) Thioredoxin and related proteins as multifunctional fusion tags for soluble expression in E. coli. Methods Mol. Biol. 205, 119-140.

Lee, J.R., Lee, S.S., Jang, H.H., Lee, Y.M., Park, J.H., Park, S.C., Moon, J.C., Park, S.K., Kim, S.Y., Lee, S.Y., Chae, H.B., Jung, Y.J., Kim, W.Y., Shin, M.R., Cheong, G.W., Kim, M.G., Kang, K.R., Lee, K.O. and Yun, D.J. (2009) Heat-shock dependent oligomeric status alters the function of a plant-specific thioredoxin-like protein, AtTDX. Proc. Natl. Acad. Sci. U S A, 106, 5978-5983.

Lee, S.B., Li, B., Jin, S. and Daniell, H. (2010) Expression and characterization of antimicrobial peptides Retrocyclin-101 and Protegrin-1 in chloroplasts to control viral and bacterial infections. Plant Biotechnol. J. 9, 100-115. 
Leelavathi, S. and Reddy, V.S. (2003) Chloroplast expression of His-tagged GUS-fusions: a general strategy to overproduce and purify foreign proteins using transplastomic plants as bioreactors. Mol. Breed. 11, 49-58.

Lemaire, S.D., Michelet, L., Zaffagnini, M., Massot, V. and Issakidis-Bourguet, E. (2007) Thioredoxins in chloroplasts. Curr. Genet. 51, 343-365.

Lentz, E.M., Segretin, M.E., Morgenfeld, M.M., Wirth, S.A., Dus Santos, M.J., Mozgovoj, M.V., Wigdorovitz, A. and Bravo-Almonacid, F.F. (2010) High expression level of a foot and mouth disease virus epitope in tobacco transplastomic plants. Planta, 231, 387-395.

Lenzi, P., Scotti, N., Alagna, F., Tornesello, M.L., Pompa, A., Vitale, A., De Stradis, A., Monti, L., Grillo, S., Buonaguro, F.M., Maliga, P. and Cardi, T. (2008) Translational fusion of chloroplast-expressed human papillomavirus type 16 L1 capsid protein enhances antigen accumulation in transplastomic tobacco. Transgenic Res. 17, 1091-1102.

McFadden, G.I. (2001) Chloroplast origin and integration. Plant Physiol. 125 50-53.

Meyer, Y., Reichheld, J.P. and Vignols, F. (2005) Thioredoxins in Arabidopsis and other plants. Photosynth. Res. 86, 419-433.

Mison, D. and Curling, J. (2000) The industrial production costs of recombinant therapeutic proteins expressed in transgenic corn. BioPharm. Int. 13, 48-54.

Mitraki, A. and King, J. (1989) Protein folding intermediates and inclusion body formation. Biotechnology, 7, 690-697.

Molina, A., Hervas-Stubbs, S., Daniell, H., Mingo-Castel, A.M. and Veramendi, J. (2004) High-yield expression of a viral peptide animal vaccine in transgenic tobacco chloroplasts. Plant Biotechnol. J. 2, 141-153.

Oey, M., Lohse, M., Kreikemeyer, B. and Bock, R. (2009) Exhaustion of the chloroplast protein synthesis capacity by massive expression of a highly stable protein antibiotic. Plant J. 57, 436-445.

Ouerghi, Z., Remy, R., Ouelhazi, L., Ayadi, A. and Brulfert, J. (2000) Twodimensional electrophoresis of soluble leaf proteins, isolated from two wheat species (Triticum durum and Triticum aestivum) differing in sensitivity towards $\mathrm{NaCl}$. Electrophoresis, 21, 2487-2491.

Park, S.K., Jung, Y.J., Lee, J.R., Lee, Y.M., Jang, H.H., Lee, S.S., Park, J.H., Kim, S.Y., Moon, J.C., Lee, S.Y., Chae, H.B., Shin, M.R., Jung, J.H., Kim, M.G., Kim, W.Y., Yun, D.J. and Lee, K.O. (2009) Heat-shock and redoxdependent functional switching of an h-type Arabidopsis thioredoxin from a disulfide reductase to a molecular chaperone. Plant Physiol. 150, 552561.

Ribas, A.V., Ho, P.L., Tanizaki, M.M., Raw, I. and Nascimento, A.L. (2000) High-level expression of tetanus toxin fragment C-thioredoxin fusion protein in Escherichia coli. Biotechnol. Appl. Biochem. 31, 91-94.
Ruhlman, T., Ahangari, R., Devine, A., Samsam, M. and Daniell, H. (2007) Expression of cholera toxin B-proinsulin fusion protein in lettuce and tobacco chloroplasts: oral administration protects against development of insulitis in non-obese diabetic mice. Plant Biotechnol. J. 5, 495-510.

Ruhlman, T., Verma, D., Samson, N. and Daniell, H. (2010) The role of heterologous chloroplast sequence elements in transgene integration and expression. Plant Physiol. 152, 2088-2104.

Sahrawy, M., Hecht, V., Lopez-Jaramillo, J., Chueca, A., Chartier, Y. and Meyer, Y. (1996) Intron position as an evolutionary marker of thioredoxins and thioredoxin domains. J. Mol. Evol. 42, 422-431.

Schurmann, P. and Buchanan, B.B. (2008) The ferredoxin/thioredoxin system of oxygenic photosynthesis. Antioxid. Redox Signal. 10, 1235-1274.

Scotti, N., Alagna, F., Ferraiolo, E., Formisano, G., Sannino, L., Buonaguro, L., De Stradis, A., Vitale, A., Monti, L., Grillo, S., Buonaguro, F.M. and Cardi, T. (2009) High-level expression of the HIV-1 Pr55gag polyprotein in transgenic tobacco chloroplasts. Planta, 229, 1109-1122.

Segui-Simarro, J.M., Testillano, P.S. and Risueno, M.C. (2003) Hsp70 and Hsp90 change their expression and subcellular localization after microspore embryogenesis induction in Brassica napus L. J. Struct. Biol. 142, 379-391.

Staub, J.M., Garcia, B., Graves, J., Hajdukiewicz, P.T., Hunter, P., Nehra, N., Paradkar, V., Schlittler, M., Carroll, J.A., Spatola, L., Ward, D., Ye, G. and Russell, D.A. (2000) High-yield production of a human therapeutic protein in tobacco chloroplasts. Nat. Biotechnol. 18, 333-338.

Xu, Z., Zhong, Z., Huang, L., Peng, L., Wang, F. and Cen, P. (2006) Highlevel production of bioactive human beta-defensin-4 in Escherichia coli by soluble fusion expression. Appl. Microbiol. Biotechnol. 72, 471-479.

Xu, X., Jin, F., Yu, X., Ren, S., Hu, J. and Zhang, W. (2007) High-level expression of the recombinant hybrid peptide cecropinA(1-8)-magainin2(112) with an ubiquitin fusion partner in Escherichia coli. Protein Expr. Purif. 55, 175-182.

Yasukawa, T., Kanei-Ishii, C., Maekawa, T., Fujimoto, J., Yamamoto, T. and Ishii, S. (1995) Increase of solubility of foreign proteins in Escherichia coli by coproduction of the bacterial thioredoxin. J. Biol. Chem. 270, 2532825331.

Ye, G.N., Hajdukiewicz, P.T., Broyles, D., Rodriguez, D., Xu, C.W., Nehra, N. and Staub, J.M. (2001) Plastid-expressed 5-enolpyruvylshikimate-3phosphate synthase genes provide high level glyphosate tolerance in tobacco. Plant J. 25, 261-270.

Yuan, S., Duan, H., Liu, C., Liu, X., Liu, T., Tao, H. and Zhang, Z. (2004) The role of thioredoxin and disulfide isomerase in the expression of the snake venom thrombin-like enzyme calobin in Escherichia coli BL21 (DE3). Protein Expr. Purif. 38, 51-60. 